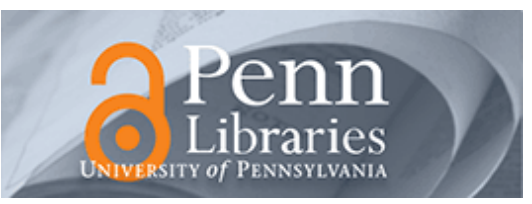

University of Pennsylvania ScholarlyCommons

\title{
Robust Model Predictive Control for Non-Linear Systems with Input and State Constraints Via Feedback Linearization
}

\author{
Yash Vardhan Pant \\ yashpant@seas.upenn.edu \\ Houssam Abbas \\ University of Pennsylvania, habbas@seas.upenn.edu \\ Rahul Mangharam \\ University of Pennsylvania, rahulm@seas.upenn.edu
}

Follow this and additional works at: https://repository.upenn.edu/mlab_papers

Part of the Computer Engineering Commons, and the Electrical and Computer Engineering Commons

\section{Recommended Citation}

Yash Vardhan Pant, Houssam Abbas, and Rahul Mangharam, "Robust Model Predictive Control for NonLinear Systems with Input and State Constraints Via Feedback Linearization", Conference on Decision and Control . January 2016.

For full version including proofs, see:

Y. V. Pant, H. Abbas, and R. Mangharam, "Tech report: Robust model predictive control for non-linear systems with input and state constraints via feedback linearization," March 2016. [Online]. Available: http://tinyurl.com/ nlrmpcwisc

This paper is posted at ScholarlyCommons. https://repository.upenn.edu/mlab_papers/94

For more information, please contact repository@pobox.upenn.edu. 


\title{
Robust Model Predictive Control for Non-Linear Systems with Input and State Constraints Via Feedback Linearization
}

\author{
Abstract \\ Robust predictive control of non-linear systems under state estimation errors and input and state \\ constraints is a challenging problem, and solutions to it have generally involved solving computationally \\ hard non-linear optimizations. Feedback linearization has reduced the computational burden, but has not \\ yet been solved for robust model predictive control under estimation errors and constraints. In this paper, \\ we solve this problem of robust control of a non-linear system under bounded state estimation errors and \\ input and state constraints using feedback linearization. We do so by developing robust constraints on \\ the feedback linearized system such that the non-linear system respects its constraints. These \\ constraints are computed at run-time using online reachability, and are linear in the optimization variables, \\ resulting in a Quadratic Program with linear constraints. We also provide robust feasibility, recursive \\ feasibility and stability results for our control algorithm. We evaluate our approach on two systems to \\ show its applicability and performance.
}

\section{Keywords}

Non-linear Control, Feedback Linearization, Predictive Control, Robust Control, Constrained System

Disciplines

Computer Engineering | Electrical and Computer Engineering

\section{Comments}

For full version including proofs, see:

Y. V. Pant, H. Abbas, and R. Mangharam, "Tech report: Robust model predictive control for non-linear systems with input and state constraints via feedback linearization," March 2016. [Online]. Available: http://tinyurl.com/nlrmpcwisc 


\title{
Robust Model Predictive Control for Non-Linear Systems with Input and State Constraints Via Feedback Linearization
}

\author{
Yash Vardhan Pant, Houssam Abbas, Rahul Mangharam
}

\begin{abstract}
Robust predictive control of non-linear systems under state estimation errors and input and state constraints is a challenging problem, and solutions to it have generally involved solving computationally hard non-linear optimizations. Feedback linearization has reduced the computational burden, but has not yet been solved for robust model predictive control under estimation errors and constraints. In this paper, we solve this problem of robust control of a non-linear system under bounded state estimation errors and input and state constraints using feedback linearization. We do so by developing robust constraints on the feedback linearized system such that the non-linear system respects its constraints. These constraints are computed at run-time using online reachability, and are linear in the optimization variables, resulting in a Quadratic Program with linear constraints. We also provide robust feasibility, recursive feasibility and stability results for our control algorithm. We evaluate our approach on two systems to show its applicability and performance.
\end{abstract}

\section{INTRODUCTION}

In this paper we are concerned with the problem of controlling nonlinear dynamical systems $S$ of the form $\dot{x}=$ $f(x)+G(x) u$ under state and input constraints, and subject to errors in the state estimate. This problem is formulated as

$$
\begin{array}{rl}
\min _{\mathbf{x}, \mathbf{u}} & l(\mathbf{x}, \mathbf{u}) \\
\text { s.t. } \dot{x} & =f(x)+G(x) u \\
x & \in X, u \in U
\end{array}
$$

where $l(\mathbf{x}, \mathbf{u})$ is a cost function whose minimization over the state and input trajectories $\mathbf{x}$ and $\mathbf{u}$ ensures stability of the system. Sets $X \subset \mathbb{R}^{n_{x}}$ and $U \subset \mathbb{R}^{n_{u}}$ encode constraints on the state (e.g., safety) and the input. The input $u=u(\hat{x})$ is a function of a state estimate that in general differs from the true state of the system.

The application of Model Predictive Control (MPC) to nonlinear systems involves the repeated solution of generally non-quadratic, non-convex optimizations. Various approaches for solving (or approximately solving) the optimizations and their trade-offs are reviewed in [1]. Another approach is to first feedback linearize the system $S$ [2]: namely, the applied control $u=u(x, v)$ is designed in such a way that the resulting closed-loop dynamics $S_{f l}$ are now linear: $S_{f l}: \dot{z}=A z+B v$. The input $v$ to the linearized dynamics can now be computed so as to optimize system performance and ensure stability. The state $z$ of the linearized

\footnotetext{
*This work was supported by STARnet a Semiconductor Research Corporation program sponsored by MARCO and DARPA, NSF MRI-0923518 and the US Department of Transportation University Transportation Center Program

The Department of Electrical and Systems Engineering, University of Pennsylvania, Philadelphia, U.S.A. \{yashpant,habbas,rahulm\}@ seas.upenn.edu
}

system $S_{f l}$ is related to the state $x$ of the nonlinear system $S$ via a (system-specific) function $T: z=T(x)$.

Previous work on nonlinear MPC with feedback linearization assumed the state $x(t)$ is perfectly known to the controller at any moment in time [3]. However in many cases, only a state estimate $\hat{x}(t)$ is available, and $\hat{x}(t) \neq x(t)$, and we handle such cases. Robust MPC (RMPC) has been investigated as a way of handling state estimation errors for linear [4] and nonlinear systems [5], [6], but not via feedback linearization. In particular, for non-linear systems, [5] develops a non-linear MPC with tube-like constraints for robust feasibility, but involves solving two (non-convex) optimal control problems. In [6], the authors solve a nonlinear Robust MPC through a bi-level optimization that involves solving a non-linear, non-smooth optimization which is challenging. [6] also guarantees a weaker form of recursive feasibility than [4] and what we guarantee in this work. In [7] the authors approximate the non-linear dynamics of a quadrotor by linearizing it around hover and apply the RMPC of [4] to the linearized dynamics. This differs significantly from our approach, where we formulate the RMPC on the feedback linearized dynamics directly, and not on the dynamics obtained via Jacobian linearization of the non-linear system. Existing work on MPC via feedback linearization and input/state constraints has also assumed that either $T$ is the identity [3], or, in the case of uncertainties in the parameters, that there are no state constraints [8]. A non-identity $T$ is problematic when the state is not perfectly known, since the state estimation error $e=\hat{x}-x$ maps to the linearized dynamics via $T$ in non-trivial ways, greatly complicating the analysis. In particular, the error bounds for the state estimate in $z$-space now depend on the current nonlinear state $x$. One of the complications introduced by feedback linearization is that the bounds on the input $(u \in U)$ may become a non-convex state-dependent constraint on the input $v$ to $S_{f l}: V=\{\underline{v}(x, U) \leq v \leq \mathbf{v}(x, U)\}$. In [3] forward reachability is used to provide inner convex approximations to the input set $V$. A non-identity $T$ increases the computational burden since the non-linear reach set must be computed (with an identity $T$, the feedback linearized reach set is sufficient).

Contributions: We develop a feedback linearization solution to the above control problem, with state estimation errors, input and state constraints, and non-identity $T$. To the best of our knowledge, this is the first feedback linearization solution to this problem. The resulting control problem is solved by RMPC with time-varying linear constraint sets.

The paper is organized as follows: in the next section we formulate the feedback linearized control problem. In Sec. 
III, we describe the RMPC algorithm we use to solve it, and prove that it stabilizes the nonlinear system. Sec. IV shows how to compute the various constraint sets involved in the RMPC formulation, and Sec. V applies our approach to an example. Sec. VI concludes the paper. An online technical report [9] contains proofs and more examples.

\section{PRoblem Formulation}

A common method for control of nonlinear systems is Feedback linearization [2]. Briefly, in feedback linearization, one applies the feedback law $u(x, v)=R(x)^{-1}(-b(x)+v)$ to (1), so that the resulting dynamics, expressed in terms of the transformed state $z=T(x)$, are linear time-invariant:

$$
S_{f l}: \dot{z}=A_{c} z+B_{c} v
$$

By using the remaining control authority in $v$ to control $S_{f l}$, we can effectively control the non-linear system for, say, stability or reference tracking. $T$ is a diffeomorphism [2] over a domain $D \subset X$. The original and transformed states, $x$ and $z$, have the same dimension, as do $u$ and $v$, i.e. $n_{x}=n_{z}$ and $n_{u}=n_{v}$. Because we are controlling the feedback linearized system, we must find constraint sets $Z$ and $V$ for the state $z$ and input $v$, respectively, such that $(z, v) \in Z \times V \Longrightarrow$ $\left(T^{-1}(z), u\left(T^{-1}(z), v\right)\right) \in X \times U$. We assume that the system (1) has no zero dynamics [2] and all states are controllable. In case there are zero dynamics, then our approach is applicable to the controllable subset of the states as long as the span of the rows of $G(x)$ is involutive [2].

For feedback linearizing and controlling (1), only a periodic state estimate $\hat{x}$ of $x$ is available. This estimate is available periodically every $\tau$ time units, so we may write $\hat{x}_{k}:=\hat{x}(k \tau)=x_{k}+e_{k}$, where $x_{k}$ and $e_{k}$ are sampled state and error respectively. We assume that $e_{k}$ is in a bounded set $E$ for all $k$. This implies that the feedback linearized system can be represented in discrete-time: $z_{k+1}=A z_{k}+B z_{k}$.

The corresponding $z$-space estimate $\hat{z}_{k}$ is $\hat{z}_{k}=T\left(\hat{x}_{k}\right)$. In general the $z$-space error $\tilde{e}_{k}:=T\left(\hat{x}_{k}\right)-T\left(x_{k}\right)$ is bounded for every $k$ but does not necessarily lie in $E$. Let $\widetilde{E}_{k}$ be the set containing $\tilde{e}_{k}$ : in Sec. IV-C we show how to compute it. Because the linearizing control operates on the state estimate and not $x_{k}$, we add a process noise term to the linearized, discrete-time dynamics. Our system model is therefore

$$
z_{k+1}=A z_{k}+B v_{k}+w_{k}
$$

where the noise term $w_{k}$ lies in the bounded set $W$ for all $k$. An estimate of $W$ can be obtained using the techniques of this paper. The problem (1) is therefore replaced by:

$$
\begin{array}{rl}
\min _{\mathbf{z}, \mathbf{v}} & q(\mathbf{z}, \mathbf{v})=\sum_{k=0}^{\infty} z_{k}^{T} Q z_{k}+v_{k}^{T} R v_{k} \\
\text { s.t. } z_{k+1} & =A z_{k}+B v_{k}+w_{k} \\
z_{k} & \in Z, v_{k} \in V, w_{k} \in W
\end{array}
$$

In general, the cost function $l(\mathbf{x}, \mathbf{u}) \neq q(\mathbf{z}, \mathbf{v})$. The objective function in (4) is a choice the control designer makes. For more details and when the quadratic form of $q(\mathbf{z}, \mathbf{v})$ is justified, see [3]. In Thm. 2, we show that minimizing this cost function, $q(\mathbf{z}, \mathbf{v})$, implies stability of the system.
It is easy to derive the dynamics of the state estimate $\hat{z}_{k}$ :

$$
\begin{aligned}
\hat{z}_{k+1} & =z_{k+1}+\tilde{e}_{k+1} \\
& =A z_{k}+B v_{k}+w_{k}+\tilde{e}_{k+1} \\
& =A \hat{z}_{k}+B v_{k}+\left(w_{k}+\tilde{e}_{k+1}-A \tilde{e}_{k}\right) \\
& =A \hat{z}_{k}+B v_{k}+\widehat{w}_{k+1}
\end{aligned}
$$

where $\widehat{w}_{k+1}=w_{k}+\tilde{e}_{k+1}-A \tilde{e}_{k}$, and lies in the set $\widehat{W}_{k+1}:=$ $W \oplus \widetilde{E}_{k+1} \oplus\left(-A \widetilde{E}_{k}\right)$.

Example 1: Consider the 2D system

$$
\dot{x}_{1}=\sin \left(x_{2}\right), \dot{x}_{2}=-x_{1}^{2}+u
$$

The safe set for $x$ is given as $X=\left\{\left|x_{1}\right| \leq \pi / 2,\left|x_{2}\right| \leq \pi / 3\right\}$, and the input set is $U=[-2.75,2.75]$. For the measurement $y=h(x)=x_{1}$, the system can be feedback linearized on the domain $D=\left\{x \mid \cos \left(x_{2}\right) \neq 0\right\}$, where it has a relative degree of $\rho=2$. The corresponding linearizing feedback input is $u=-\tan \left(x_{2}\right)+\left(\cos \left(x_{2}\right)\right) v$. The feedback linearized system is $\dot{z}_{1}=z_{2}, \dot{z}_{2}=v$, where $T$ is given by $z=T\left(\left(x_{1}, x_{2}\right)\right)=$ $\left(x_{1}, \sin \left(x_{2}\right)\right)$. We can analytically compute the safe set in $z$-space as $Z=T(X)=\left\{\left|z_{1}\right| \leq \pi / 2,\left|z_{2}\right| \leq 0.8660\right\}$.

For a more complicated $T$, it is not possible to obtain analytical expressions for $Z$. The computation of $Z$ in this more general case is addressed in the online appendix [9].

Notation. Given two subsets $A, B$ of $\mathbb{R}^{n}$, their Minkowski sum is $A \oplus B:=\{a+b \mid a \in A, b \in B\}$. Their Pontryagin difference is $A \ominus B=\left\{c \in \mathbb{R}^{n} \mid c+b \in A \forall b \in B\right\}$. Given integers $n \leq m,[n: m]:=\{n, n+1, \ldots, m\}$.

Assumption. Our approach applies when $X, U, E$ and $W$ are arbitrary convex polytopes (i.e. bounded intersections of half-spaces). For the sake of simplicity, in this paper we assume they are all hyper-rectangles that contain the origin

\section{RoBUST MPC FOR THE FEEDBACK LINEARIZED SYSTEM}

Following [4], [10], we formulate a Robust MPC (RMPC) controller of (4) via constraint restriction. We outline the idea before providing the technical details. The key idea is to move the effects of estimation error $\tilde{e}_{k}$ and process noise $w_{k}$ (the 'disturbances') to the constraints, and work with the nominal (i.e., disturbance-free) dynamics: $\bar{z}_{k+1}=$ $A \bar{z}_{k}+B v_{k}, \bar{z}_{0}=\hat{z}_{0}$. Because we would be optimizing over disturbance-free states, we must account for the noise in the constraints. Specifically, rather than require the next (nominal) state $\bar{z}_{k+1}$ to be in $Z$, we require it to be in the shrunk set $Z \ominus \widehat{W}_{k+1 \mid k} \ominus \widetilde{E}_{k+1 \mid k}$ : by definition of Pontryagin difference, this implies that whatever the actual value of the noise $\widehat{w}_{k+1} \in \widehat{W}_{k+1 \mid k}$ and of the estimation error $\tilde{e}_{k+1} \in$ $\widetilde{E}_{k+1 \mid k}$, the actual state $z_{k+1}$ will be in $Z$. This is repeated over the entire MPC prediction horizon $j=1, \ldots, N$, with further shrinking at every step. For further steps $(j>1)$, the process noise $\widehat{w}_{k+j \mid k}$ is propagated through the dynamics, so the shrinking term $\widehat{W}$ is shaped by a stabilizing feedback controller $\bar{z} \mapsto K \bar{z}$. At the final step $(j=N+1)$, a terminal constraint is derived using the worst case estimation error set $\widetilde{E}_{\max }$ and a global inner approximation for the input constraints, $V_{\text {inner-global }}$. 
Through this successive constraint tightening we ensure robust safety and feasibility of the feedback linearized system (and hence of the non-linear system). Since we use just the nominal dynamics, and show that the tightened constraints are linear in the state and inputs, we still solve a Quadratic Program (QP) for the RMPC optimization. The difficulty of applying RMPC in our setting is that the amounts by which the various sets are shrunk vary with time because of the time-varying state estimation error, are state-dependent, and involve set computations with the non-convexity preserving mapping $T$. One of our contributions in this paper is to establish recursive feasibility of RMPC with time-varying constraint sets.

The RMPC optimization $\mathbb{P}_{k}\left(\hat{z}_{k}\right)$ for solving (4) is:

$$
\begin{aligned}
J^{*}\left(\bar{z}_{k}\right)= & \min _{\overline{\mathbf{z}}, \mathbf{u}} \sum_{j=0}^{N}\left\{\bar{z}_{k+j \mid k}^{T} Q \bar{z}_{k+j \mid k}+v_{k+j \mid k}^{T} R v_{k+j \mid k}\right\} \\
& +\bar{z}_{k+N+1 \mid k}^{T} Q_{f} \bar{z}_{k+N+1 \mid k} \\
\bar{z}_{k \mid k}= & \hat{z}_{k} \\
\bar{z}_{k+j+1 \mid k}= & A \bar{z}_{k+j \mid k}+B v_{k+j \mid k}, j=0, \ldots, N \\
\bar{z}_{k+j \mid k} \in & \bar{Z}_{k+j \mid k}, j=0, \ldots, N \\
v_{k+j \mid k} \in & V_{k+j \mid k}, j=0, \ldots, N-1 \\
p_{N+1}= & {\left[z_{k+N+1 \mid k}, v_{k+N \mid k}\right]^{T} \in P_{f} }
\end{aligned}
$$

Here, $\bar{z}$ is the state of the nominal feedback linearized system. The cost and constraints of the optimization are explained below:

- Eq. (7a) shows a cost quadratic in $\bar{z}$ and $v$, where as usual $Q$ is positive semi-definite and $R$ is positive definite. In the terminal cost term, $Q_{f}$ is the solution of the Lyapunov equation $Q_{f}-(A+B K)^{T} Q_{f}(A+B K)=$ $Q+K^{T} R K$. This choice guarantees that the terminal cost equals the infinite horizon cost under a linear feedback control $\bar{z} \mapsto K \bar{z}[11]$.

- Eq. (7b) initializes the nominal state with the current state estimate.

- Eq. (7c) gives the nominal dynamics of the discretized feedback linearized system.

- Eq. (7d) tightens the admissible set of the nominal state by a sequence of shrinking sets.

- Eq. (7e) constrains $v_{k+j \mid k}$ such that the corresponding $u(x, v)$ is admissible, and the RMPC is recursively feasible.

- Eq. (7f) constrains the final input and nominal state to be within a terminal set $P_{f}$.

The details of these sets' definitions and computations are given in Sec. IV.

\section{A. State and Input Constraints for the Robust MPC}

The state and input constraints for the RMPC are defined as follows:

The state constraints $\bar{Z}_{k+j \mid k}$ : The tightened state constraints are functions of the error sets $\widetilde{E}_{k+j \mid k}$ and disturbance sets $\widehat{W}_{k+j \mid k}$, and defined $\forall j=0, \ldots, N$

$$
\bar{Z}_{k+j \mid k}=Z \ominus_{i=0}^{j-1}\left(L_{i} \widehat{W}_{k+(j-i) \mid k}\right) \ominus\left(-\widetilde{E}_{k+j \mid k}\right)
$$

(Recall $Z$ is a subset of $T(X), \widehat{W}_{k+j \mid k}$ and $\widetilde{E}_{k+j \mid k}$ bound the estimation error and noise, resp., and are formally defined in Sec. IV). The state transition matrix $L_{j}, \forall j=0, \ldots, N$ is defined as $L_{0}=\mathbb{I}, L_{j+1}=(A+B K) L_{j}$. The intuition behind this construction was given at the start of this section.

The input constraints $V_{k+j \mid k}: \forall j=0, \ldots, N-1$

$$
V_{k+j \mid k}=\underline{V}_{k+j \mid k} \ominus_{i=0}^{j-1} K L_{i} \widehat{W}_{k+(j-i) \mid k}
$$

where $\underline{V}_{k+j \mid k}$ is an inner-approximation of the set of admissible inputs $v$ at prediction step $j+k \mid k$, as defined in Sec. IV-B. The intuition behind this construction is similar to that of $\bar{Z}_{k+j \mid j}$ : given the inner approximation $\underline{V}_{k \mid k}$, it is further shrunk at each prediction step $j$ by propagating forward the noise $\widehat{w}_{k}$ through the dynamics, and shaped according to the stabilizing feedback law $K$, following [4].

The terminal constraint $P_{f}$ : This constrains the extended state $p_{k}=\left[\bar{z}_{k}, v_{k-1}\right]^{T}$, and is given by

$$
P_{f}=C_{p} \ominus\left[\begin{array}{c}
(A+B K)^{N} \\
K(A+B K)^{N-1}
\end{array}\right] \widehat{W}_{\max }
$$

where $\widehat{W}_{\max } \subset \mathbb{R}^{n_{z}}$ is a bounding set on the worst-case disturbance (we show how it's computed in Sec. IV-C), and $C_{p} \subset \mathbb{R}^{n_{z}} \times \mathbb{R}^{n_{v}}$ is an invariant set of the nominal dynamics subject to the stabilizing controller $\bar{z} \mapsto K \bar{z}$, naturally extended to the extended state $p$ : that is, there exists a feedback control law $p \mapsto \widehat{K} p$, such that $\forall p \in C_{p}$

$$
\widehat{A} p+\widehat{B} \widehat{K} p+\widehat{L}_{N}\left[\widehat{w}^{T}, 0^{T}\right]^{T} \in C_{p}, \forall \widehat{w} \in \widehat{W}_{\max }
$$

with $\widehat{A}=\left[\begin{array}{cc}A & 0_{n \times m} \\ 0_{m \times n} & 0_{m \times m}\end{array}\right], \widehat{B}=\left[\begin{array}{c}B \\ \mathbb{I}_{m \times m}\end{array}\right], \widehat{K}=$ $\left[\begin{array}{ll}K & 0_{m \times m}\end{array}\right], \widehat{L}_{N}=(\widehat{A}+\widehat{B} \widehat{K})^{N}$. It is important to note the following:

- The set $P_{f}$ can be computed offline since it depends on $\widehat{W}_{\max }, \widetilde{E}_{\max }$ and the global inner approximation for the constraints on $v, V_{\text {inner-global }}$, all of which can be computed offline.

- If $P_{f}$ is non-empty, then all intermediate sets that appear in (7) are also non-empty, since $P_{f}$ shrinks the state and input sets by the maximum disturbances $\widehat{W}_{\max }$ and $\widetilde{E}_{\max }$. Thus we can tell, before running the system, whether RMPC might be faced with empty constraint sets (and thus infeasible optimizations).

- Note that all constraints are linear.

\section{B. The Control Algorithm}

We can now describe the algorithm used for solving (7) by robust receding horizon control.

\section{Robust Feasibility and Stability}

We are now ready to state the main result of this paper: namely, that the RMPC of the feedback linearized system (7) is feasible at all time steps if it starts out feasible, and that it stabilizes the nonlinear system, for all possible values of the state estimation error and feedback linearization error.

Theorem 1 (Robust Feasibility): If at some time step $k_{0} \geq 0$, the RMPC optimization $\mathbb{P}_{k_{0}}\left(\hat{z}_{k_{0}}\right)$ is feasible, then all subsequent optimizations $\mathbb{P}_{k}\left(\hat{z}_{k}\right) k>k_{0}$ are also feasible. 


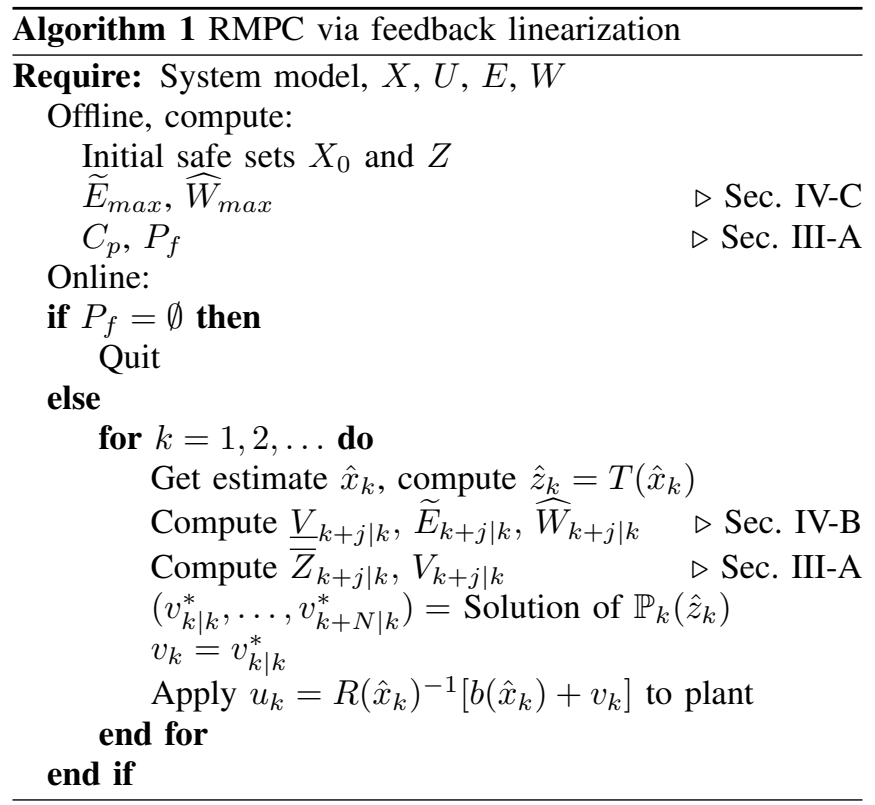

Moreover, the nonlinear system (1) controlled by algorithm 1 and subject to the disturbances $(E, W)$ satisfies its state and input constraints at all times $k \geq k_{0}$.

Theorem 2 (Stability): Given an equilibrium point $x_{e} \in$ $X_{0} \subset T^{-1}(Z)$ of the nonlinear dynamics (1), Algorithm 1 stabilizes the nonlinear system to an invariant set around $x_{e}$. The proofs are in the online report [9].

\section{SET DEFinitions FOR THE RMPC}

Algorithm 1 and the problem $\mathbb{P}_{k}\left(\hat{z}_{k}\right)$ (7) use a number of constraint sets to ensure recursive feasibility of the successive RMPC optimizations, namely: inner approximations of the admissible input sets $\underline{V}_{k+j \mid k}$, bounding sets for the (Tmapped) estimation error $\widetilde{E}_{k+j \mid k}$, bounding sets for the process noise $\widehat{W}_{k+j \mid k}$, and the largest error and noise sets $\widetilde{E}_{\text {max }}$ and $\widehat{W}_{\text {max }}$. In this section we show how these sets are defined and computed. Note, our approach is an extension to [3] as: 1) we compute the feasible set for the states of the feedback linearized system under a non-trivial diffeomorpism $T, 2$ ) we compute the bounding sets for disturbances while considering estimation error and process noise, neither of which are considered in [3]. In addition, due to the presence of state-estimation error, we compute these sets using an over-approximation of the reach set, as seen in the following subsection.

Since we control the system in $z$-space, we need to compute a set $Z \subset \mathbb{R}^{n_{z}}$ s.t. $z \in Z \Longrightarrow x=T^{-1}(z) \in$ $X$. Moreover, to check feasibility at time 0 of the MPC optimization, we need a subset $X_{0} \subset X$ s.t. $x \in X_{0} \Longrightarrow$ $z=T(x) \in Z$. Mapping sets between $z$ and $x$ spaces via the arbitrary diffeomorphism $T$ has to be done numerically, and we show how in the online report [9].

\section{A. Approximating the reach set of the nonlinear system}

First we show how to compute an outer-approximation of the $j$-step reach set of the nonlinear system, starting at time $k, \mathbf{X}_{k+j \mid k}$. This is needed for computing $\underline{V}_{k+j \mid k}$ and $\widetilde{E}_{k+j \mid k}$.

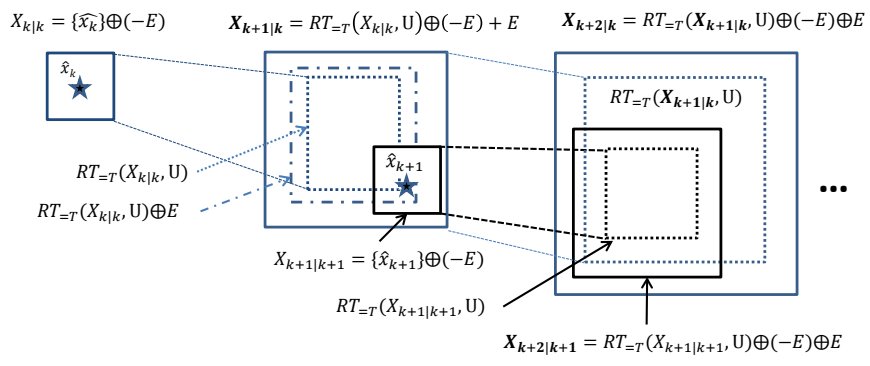

Fig. 1. The outer-approximated reach sets for $x_{k+j}$, computed at time steps $k, k+1$, used to compute $\widetilde{E}_{k+j \mid k}, \underline{V}_{k+j \mid k}$.

In all but the simplest systems, forward reachable sets cannot be computed exactly. To approximate them we may use a reachability tool for nonlinear systems like RTreach [12]. A reachability tool computes an outer-approximation of the reachable set of a system starting from some set $\mathcal{X} \subset X$, subject to inputs from a set $U$, for a duration $T \geq 0$. Denote this approximation by $R T_{T}(\mathcal{X}, U)$, so $x(T) \in R T_{T}(\mathcal{X}, U)$ for all $T, x(0) \in \mathcal{X}$ and $u:[0, T] \rightarrow U$.

At time $k$, the state estimate $\hat{x}_{k}$ is known. Therefore $x_{k}=$ $\hat{x}_{k}-e_{k} \in \hat{x}_{k} \oplus(-E):=X_{k \mid k}$. Propagating $X_{k \mid k}$ forward one step through the continuous-time nonlinear dynamics yields $X_{k+1 \mid k}$, which is outer-approximated by $R T_{T}\left(X_{k \mid k}, U\right)$. The state estimate that the system will receive at time $k+1$ is therefore bound to be in the set $R T_{T}\left(X_{k \mid k}, U\right) \oplus E$. Since $0 \in E$, we maintain $X_{k+1 \mid k} \subset R T_{T}\left(X_{k \mid k}, U\right) \oplus E$. For $1 \leq j \leq N$, we define the $j$-step over-approximate reach set computed at time $k$ to be

$$
\begin{aligned}
\mathbf{X}_{k \mid k} & :=\hat{x}_{k} \oplus(-E) \\
\mathbf{X}_{k+j \mid k} & :=R T_{T}\left(\mathbf{X}_{k+j-1 \mid k}, U\right) \oplus E \oplus(-E)
\end{aligned}
$$

(The reason for adding the extra $-E$ term will be apparent in the proof to Thm. 1). Fig. 1 shows a visualization of this approach. The following holds by construction:

Lemma 3: For any time $k$ and step $j \geq 1, X_{k+j \mid k} \subset$ $\mathbf{X}_{k+j \mid k}$.

This construction of $\mathbf{X}_{k+j \mid k}$ permits us to prove recursive feasibility of the RMPC controller, because it causes the constraints of the RMPC problem setup at time $k+1$ to be consistent with the constraints of the problem setup at time $k$.

\section{B. Approximating the bounding sets for the input}

Given $x \in X$, define the set $V(x):=\left\{v \in \mathbb{R}^{n_{v}} \mid u(x)=\right.$ $\left.R^{-1}(x)[b(x)+v] \in U\right\}$. We assume that there exist functions $\underline{v}_{i}, \mathbf{v}_{i}: X \rightarrow \mathbb{R}$ s.t. for any $x, V(x)=$ $\left\{\left[v_{1}, \ldots, v_{n_{v}}\right]^{T} \mid \underline{v}_{i}(x ; U) \leq v_{i} \leq \mathbf{v}_{\mathbf{i}}(x ; U)\right\}$. Because in general $V(x)$ is not a rectangle, we work with inner and outer rectangular approximations of $V(x)$. Specifically, let $\mathcal{X}$ be a subset of $X$. Define the inner and outer bounding rectangles, respectively

$$
\begin{aligned}
& \underline{V}(\mathcal{X}):=\left\{\left[v_{1}, \ldots, v_{n_{v}}\right]^{T} \mid \max _{x \in \mathcal{X}} \underline{v}_{i}(x ; U) \leq v_{i} \leq \min _{x \in \mathcal{X}} \mathbf{v}_{i}(x ; U)\right\} \\
& \mathbf{V}(\mathcal{X}):=\left\{\left[v_{1}, \ldots, v_{n_{v}}\right]^{T} \mid \min _{x \in \mathcal{X}} \underline{v}_{i}(x ; U) \leq v_{i} \leq \max _{x \in \mathcal{X}} \mathbf{v}_{i}(x ; U)\right\}
\end{aligned}
$$

By construction, we have for any subset $\mathcal{X} \subset X$

$$
\underline{V}(\mathcal{X}) \subseteq \cap_{x \in \mathcal{X}} V(x) \subset \mathbf{V}(\mathcal{X})
$$




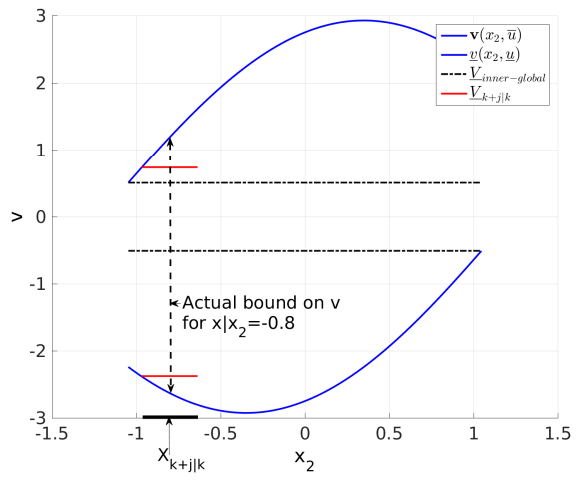

Fig. 2. Local and global inner approximations of input constraints for running example, with $\mathbf{X}_{k+j \mid k}=[-\pi / 4,0] \times[-0.9666,-0.6283]$ for some $k, j$ and $U=[-2.75,2.75]$. Color in online version.

If two subsets of $X$ satisfy $\mathcal{X}_{1} \subset \mathcal{X}_{2}$, then it holds that

$$
\underline{V}\left(\mathcal{X}_{2}\right) \subset \underline{V}\left(\mathcal{X}_{1}\right), \mathbf{V}\left(\mathcal{X}_{1}\right) \subset \mathbf{V}\left(\mathcal{X}_{2}\right)
$$

We can compute:

$$
\underline{V}_{k+j \mid k}=\underline{V}\left(\mathbf{X}_{k+j \mid k}\right), \underline{V}_{\text {inner-global }}=\underline{V}(X)
$$

In practice we use interval arithmetic to compute these sets since $\mathbf{X}_{k+j \mid k}$ and $U$ are hyper-intervals. Fig. 2 shows these sets for the running example.

\section{Approximating the bounding sets for the disturbances}

We will also need to define containing sets for the state estimation error in $z$ space: recall that $\hat{z}_{k}=T\left(\hat{x}_{k}\right)=T\left(x_{k}+\right.$ $e_{k}$ ). We use a Taylor expansion (and the remainder theorem)

$$
\begin{aligned}
\hat{z}_{k} & =T\left(x_{k}\right)+\underbrace{\frac{d T}{d x}\left(x_{k}\right)}_{M\left(x_{k}\right)} e_{k}+\underbrace{\frac{1}{2} e_{k}^{T} \frac{d^{2} T}{d x^{2}}(c) e_{k}}_{r_{k}(c)}, c \in x_{k}+E \\
& =T\left(x_{k}\right)+M\left(x_{k}\right) e_{k}+r_{k}(c), c \in x_{k}+E \\
& =T\left(x_{k}\right)+h_{k}+r_{k}(c), c \in x_{k}+E
\end{aligned}
$$

The remainder term $r_{k}(c)$ is bounded in the set

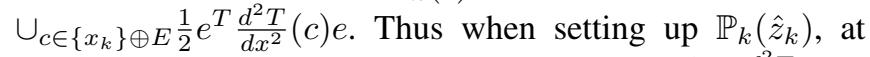
the $j^{t h}$ step, $r_{k+j \mid k} \in D_{k+j \mid k}:=\cup_{c \in \mathbf{X}_{k+j \mid k} \oplus E} \frac{1}{2} e^{T} \frac{d^{2} T}{d x^{2}}(c) e$, where $\mathbf{X}_{k+j \mid k}$ is the reach set computed in (12).

The error $h_{k}$ lives in $\cup_{x \in X_{k}, e \in E} M(x) e$. Thus when setting up $\mathbb{P}_{k}\left(\hat{z}_{k}\right)$, the error $h_{k+j \mid k}$ lives in $\cup_{x \in \mathbf{X}_{k+j \mid k}} M(x) E$. Finally the rectangular over-approximation of this set is

$$
\begin{array}{r}
H_{k+j \mid k}=\left\{h \mid \sum_{\ell=1}^{n_{x}} \min _{x \in \mathbf{X}_{k+j \mid k}, e \in E} M_{i \ell}(x) e(\ell) \leq h(i)\right. \\
\left.\leq \sum_{\ell=1}^{n_{x}} \max _{x \in \mathbf{X}_{k+j \mid k}, e \in E} M_{i \ell}(x) e(\ell)\right\}
\end{array}
$$

where $M_{i \ell}$ is the $(i, \ell)^{t h}$ element of matrix $M$ and $h(\ell)$ is the $\ell^{\text {th }}$ element of $h$.

Therefore the state estimation error $h_{k+j \mid k}+r_{k+j \mid k}$ is bounded in the set $H_{k+j \mid k} \oplus D_{k+j \mid k}$. In the experiments we ignore the remainder term $D_{k+j \mid k}$ based on the observation that $e_{k}$ is small relative to the state $x_{k}$. Thus we use:

$$
\widetilde{E}_{k+j \mid k}=H_{j+k \mid j}
$$

Example 2: For the running example (6), we have $M=$ $\left[1,0 ; 0, \cos \left(x_{2}\right)\right]$. If the estimation error $e$ (in radians) is bounded in $E=\left\{e\|\| e \|_{\infty} \leq 0.0227\right\}$, then the relative linearization error, averaged over several realizations of the error, is less than $2 \cdot 10^{-3}$.

We also need to calculate containing sets for the process noise $\widehat{w}$. Recall that for all $k, j, \hat{z}_{k+j+1}=A \hat{z}_{k+j}+B v_{k}+$ $\widehat{w}_{k+j+1}$. Therefore

$$
\widehat{w}_{k+j+1} \in \widehat{W}_{k+j+1 \mid k}:=W \oplus \widetilde{E}_{k+j+1 \mid k} \oplus\left(-A \widetilde{E}_{k+j \mid k}\right)
$$

We also define the set $\widetilde{E}_{\max }$, which is necessary for the terminal constraints of Eq. (10). $\tilde{E}_{\max }$ represents the worst case bound on the estimation error $\tilde{e}_{k}$, and is computed similar to Eq. (16), but over the entire set $X$.

$\widehat{W}_{\max }$ is then defined as:

$$
\widehat{W}_{\max }=W \oplus \tilde{E}_{\max } \oplus\left(-A \tilde{E}_{\max }\right)
$$

\section{EXPERIMENTS}

We evaluate our approach on a 4D flexible joint manipulator and a simple 2-state example (in the online technical report [9]). We implemented the RMPC controller of Alg. 1 in MATLAB The set computations were done using the MPT Toolbox [13], and the invariant set computations using the Matlab Invariant Set Toolbox [14]. The reachability computations for $\mathbf{X}_{k+j \mid k}$ were performed on the linear dynamics and mapped back to $x$-space. The RMPC optimizations were formulated in CVX [15] and solved by Gurobi [16].

\section{A. Single link flexible joint manipulator}

We consider the single link flexible manipulator system $S$, also used in [8] and [17] whose dynamics are given by:

$$
S:\left[\begin{array}{c}
\dot{x}_{1} \\
\dot{x}_{2} \\
\dot{x}_{3} \\
\dot{x}_{4}
\end{array}\right]=\left[\begin{array}{c}
x_{2} \\
-\frac{m g l}{I} \sin \left(x_{1}\right)-\frac{\sigma}{I}\left(x_{1}-x_{3}\right) \\
x_{4} \\
\frac{\sigma}{J}\left(x_{1}-x_{3}\right)
\end{array}\right]+\left[\begin{array}{c}
0 \\
0 \\
0 \\
\frac{1}{J}
\end{array}\right] u
$$

This models a system where a motor, with an angular moment of inertia $J=1$, is coupled to a uniform thin bar of mass $m=1 / g$, length $l=1 \mathrm{~m}$ and moment of inertia $I=1$, through a flexible torsional string with stiffness $\sigma=1$ and $g=9.8 \mathrm{~ms}^{-2}$. States $x_{1}$ and $x_{2}$ are the angles of the bar and motor shaft in radians, respectively, and $x_{3}, x_{4}$ are their respective rotational speeds in radians/sec. The safe set is the box $X=[-\pi / 4, \pi / 4] \times[-\pi / 4, \pi / 4] \times[-\pi, \pi] \times[-\pi, \pi]$. The input torque $u$ is bounded in $U=[\underline{u}, \bar{u}]=[-10,10] N$. $m$. The estimation error $e=\hat{x}-x$ is bounded in $E=$ $[-\pi / 180, \pi / 180]^{4} \in \mathbb{R}^{4}$ and $W=\left[-10^{-3}, 10^{-3}\right]^{4} \in \mathbb{R}^{4}$.

The diffeomorphism $T$ is given by:

$$
z=T(x)=\left[\begin{array}{c}
x_{1} \\
x_{2} \\
-\frac{m g l}{I} \sin \left(x_{1}\right)-\frac{\sigma}{I}\left(x_{1}-x_{3}\right) \\
\frac{m g l}{I} x_{2} \cos \left(x_{1}\right)-\frac{\sigma}{I}\left(x_{2}-x_{4}\right)
\end{array}\right]
$$

The input to the feedback linearized system is given by $v=\beta u+\alpha(x)$ where $\beta=\frac{\sigma}{I J}$ and $\alpha(x)=\frac{m g l}{I} x_{2}^{2} \sin \left(x_{1}\right)+$ $\frac{\sigma^{2}}{I J}\left(x_{1}-x_{3}\right)-\left(\frac{m g l}{I} \cos \left(x_{1}\right)-\frac{\sigma}{I}\right)\left(\frac{m g l}{I} \sin \left(x_{1}\right)+\frac{\sigma}{I}\left(x_{1}-x_{3}\right)\right)$ The feedback linearized system $S_{f l}$ has the dynamics: $\dot{z}_{1}=$ $z_{2}, \dot{z_{2}}=z_{3}, \dot{z_{3}}=z_{4}, \dot{z_{4}}=v$. 

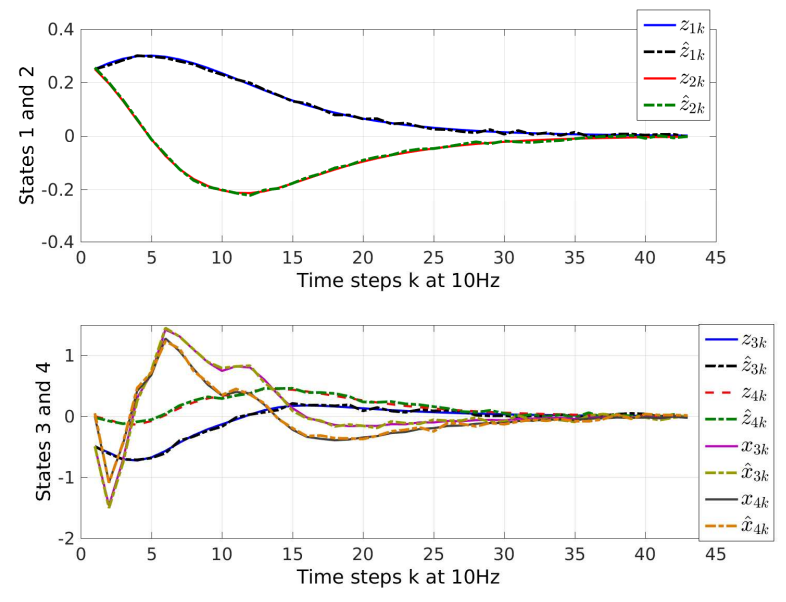

Fig. 3. The states and their estimates of the feedback linearized and nonlinear manipulator. Note $z_{1}=x_{1}$ and $z_{2}=x_{2}$. Color in online version.

A global inner approximation of the $v$ input set is computed, via interval arithmetic, as $V_{\text {inner-global }}=$ $\left[\max _{x \in X} \alpha(x)+\beta \underline{u}, \min _{x \in X} \alpha(x)+\beta \bar{u}\right]$. Similarly, the inner approximations $\underline{V}_{k+j \mid k}$ are computed online by interval arithmetic as $\underline{V}_{k+j \mid k}=\left[\max _{x \in \mathbf{X}_{k+j \mid k}} \alpha(x)+\right.$ $\left.\beta \underline{u}, \min _{x \in \mathbf{X}_{k+j \mid k}} \alpha(x)+\beta \bar{u}\right]$. Using the procedure in the appendix [9] the set of safe states for $S_{f l}$ is given by $Z=$ $[-0.5121,0.5121]^{2} \times[-2.5347,2.5347] \times[-2.5603,2.5603]$. Also $X_{0}=[-0.4655,0.4655]^{2} \times[-2.7598,2.7598] \times$ $[-2.793,2.793]$. Comparing it to the set $X$, it shows that we can stabilize the system starting from initial states in a significantly large region in $X$.

We applied our controller to the above system with a discretization rate of $10 \mathrm{~Hz}$ and MPC horizon $N=10$. Fig.3 show the states of the feedback linearized system $S_{f l}$. They converge to the origin in the presence of estimation error, while respecting all constraints. Fig. 3 also shows $x_{3}$ and $x_{4}$ : they also converge to zero. Fig. 4 shows the input $v$ to $S_{f l}$ along with the global inner approximation $V_{\text {inner-global }}$ and the $x$-dependent inner approximations at the instant when the control is applied, $\underline{V}_{k \mid k}$ computed online. Note that the bounds computed online allow for significantly more control action compared to the conservative global inner approximation. Finally, Fig. 4 also shows the input $u$ applied to the non-linear system (and its bounds), which robustly respects its constraints $u \in U$.

\section{DISCUSSION}

In this paper we develop the first algorithm for robust control of a non-linear system with estimation errors and state and input constraints via feedback linearization and Robust MPC. Experimental results show that the control algorithm stabilizes the systems while ensuring robust constraint satisfaction. While we only evaluated our approach for single input systems, the formulation and set computations involved hold as is for multi-input systems as well.

Limitations of the approach mostly have to do with the numerical limitations involved in computing the constraint sets, and potential conservatism of the approximations.
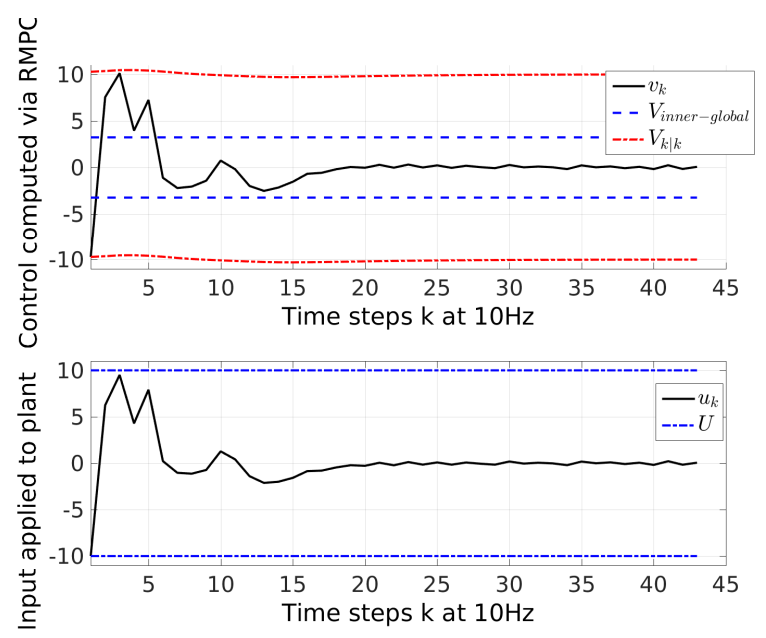

Fig. 4. Inputs $v$ and $u$ and their bounds for the manipulator example. Color in online version.

\section{REFERENCES}

[1] M. Cannon, "Efficient nonlinear model predictive control algorithms," Annual Reviews in Control, vol. 28, no. 2, pp. 229 - 237, 2004.

[2] H. Khalil, "Nonlinear systems," in Prentice Hall, 2002.

[3] D. Simon, J. Lofberg, and T. Glad, "Nonlinear model predictive control using feedback linearization and local inner convex constraint approximations," in Control Conference (ECC), 2013 European, July 2013, pp. 2056-2061.

[4] A. Richards and J. How, "Robust model predictive control with imperfect information," in American Control Conference, 2005. Proceedings of the 2005, June 2005, pp. 268-273.

[5] D. Mayne and E. Kerrigan, "Tube-based Robust Nonlinear Model Predictive Control ," in IFAC Symposium on Nonlinear Control Systems, 2007.

[6] S. Streif, M. Kogel, T. Bathge, and R. Findeisen, "Robust Nonlinear Model Predictive Control with Constraint Satisfaction: A Relaxationbased Approach," in IFAC World Congress, 2014.

[7] W. Zhao and T. H. Go, "Quadcopter formation flight control combining mpc and robust feedback linearization," Journal of the Franklin Institute, vol. 351, no. 3, pp. 1335 - 1355, 2014.

[8] W. Son, J. Choi, and O. Kwon, "Robust control of feedback linearizable system with the parameter uncertainty and input constraint," in Proceedings of the 40th SICE Annual Conference, 2001.

[9] Y. V. Pant, H. Abbas, and R. Mangharam, "Tech report: Robust model predictive control for non-linear systems with input and state constraints via feedback linearization," March 2016. [Online]. Available: http://tinyurl.com/nlrmpcwisc

[10] Y. V. Pant, K. Mohta, H. Abbas, T. X. Nghiem, J. Devietti, and R. Mangharam, "Co-design of anytime computation and robust control," in RTSS, Dec 2015, pp. 43-52.

[11] B. Kouvaritakis and M. Cannon, Model Predictive Control: Classical, Robust and Stochastic. Springer Verlag, 2015.

[12] T. T. Johnson, S. Bak, M. Caccamo, and L. Sha, "Real-time reachability for verified simplex design," ACM Trans. Embed. Comput. Syst., vol. 15, no. 2, pp. 26:1-26:27, Feb. 2016. [Online]. Available: http://doi.acm.org/10.1145/2723871

[13] M. Herceg, M. Kvasnica, C. Jones, and M. Morari, "Multi-Parametric Toolbox 3.0," in Proc. of the ECC, Zürich, Switzerland, July 17-19 2013, pp. 502-510, http://control.ee.ethz.ch/ mpt.

[14] E. Kerrigan, "Matlab invariant set toolbox version 0.10.5," 2016. [Online]. Available: \{http://www-control.eng.cam.ac.uk/eck21/matlab/ invsetbox

[15] M. Grant and S. Boyd, "CVX: Matlab software for disciplined convex programming, version 2.0 beta," http://cvxr.com/cvx, Sep. 2013.

[16] I. Gurobi Optimization, "Gurobi optimizer reference manual," 2015. [Online]. Available: http://www.gurobi.com

[17] M. Seidi, M. Hajiaghamemar, and B. Segee, "Fuzzy Control Systems: LMI-Based Design," Fuzzy Controllers- Recent Advances in Theory and Applications, InTech, 2012. 